\title{
Les réseaux « asiatiques » et les missionnaires vannetais de la Société des Missions Étrangères de Paris au XVIII ${ }^{\mathrm{e}}$ siècle
}

\section{Évelyne Hiet-Guihur}

\section{(C) OpenEdition}

\section{Journals}

\section{Édition électronique}

URL : http://journals.openedition.org/abpo/490

DOI : $10.4000 / a b p o .490$

ISBN : 978-2-7535-1515-4

ISSN : 2108-6443

\section{Éditeur}

Presses universitaires de Rennes

Édition imprimée

Date de publication : 30 octobre 2009

Pagination : 23-42

ISBN : 978-2-7535-1008-1

ISSN : 0399-0826

\section{Référence électronique}

Évelyne Hiet-Guihur, "Les réseaux « asiatiques » et les missionnaires vannetais de la Société des Missions Étrangères de Paris au XVIII e siècle », Annales de Bretagne et des Pays de l'Ouest [En ligne], 116-3 | 2009, mis en ligne le 30 octobre 2011, consulté le 01 mai 2019. URL : http:// journals.openedition.org/abpo/490; DOI : 10.4000/abpo.490 


\title{
Les réseaux " asiatiques" et les missionnaires vannetais de la Société des Missions Étrangères de Paris au XVIII siècle *
}

\author{
Évelyne HIET-GUIHUR \\ Doctorante \\ CERHIO UMR 6258 - université de Bretagne Sud
}

Le Dictionnaire de Trévoux (1771) définit comme suit le terme de " vocation ": la vocation est la divinus afflatus, un " souffle divin ". C'est la grâce que Dieu fait quand il appelle quelqu'un à lui, et le met dans le chemin du salut ${ }^{1}$. Mais la vocation est aussi une destination à un état ou à une profession. Dans les deux cas, selon ces définitions, s'il s'agit " de vraies vocations, [celles-ci] sont vocations de Dieu, puisque c'est à lui de [...] placer tous [les individus] comme il lui plaît ${ }^{2}$ ".

La vocation ne concerne donc pas seulement l'état ecclésiastique. En effet, l'idée que la société représente l'ordre divin sur terre est présente dans l'ensemble de l'organisation sociale. En revanche, la vocation est réelle chez un grand nombre de novices et de candidats aux ordres durant l'Ancien Régime. Nous pensons ici aux propos de Jean de Viguerie qui insiste sur l'existence de vraies vocations durant l'Ancien Régime et nuance l'importance des "vocations forcées ${ }^{3}$ ".

* Cet article est issu d'une communication orale présentée en avril 2007 à l'université du Maine lors d'une journée d'étude organisée par Hervé Guillemain et consacrée aux vocations religieuses et laïques.

1. Dictionnaire universel françois et latin, vulgairement appelé Dictionnaire de Trévoux : contenant la signification et la définition tant des mots de l'une et l'autre langue..., $6^{\mathrm{e}}$ édition, reproduction de l'édition de Paris par la Compagnie des libraires associés, 1771, notice "vocation ", vol 8, p. 447-448.

2. Ibidem, p. 447.

3. VIGUERIE, Jean de, " La Vocation sacerdotale et religieuse aux XVII et XVIII ${ }^{\mathrm{e}}$ siècles. La théorie et la réalité ", dans rencontre d'histoire religieuse, $2^{\mathrm{e}}$, 1978, La Vocation religieuse et sacerdotale en France XVIT'-XIX ${ }^{e}$ siècles, Angers, Université d'Angers, 1979, p. 27-39. 

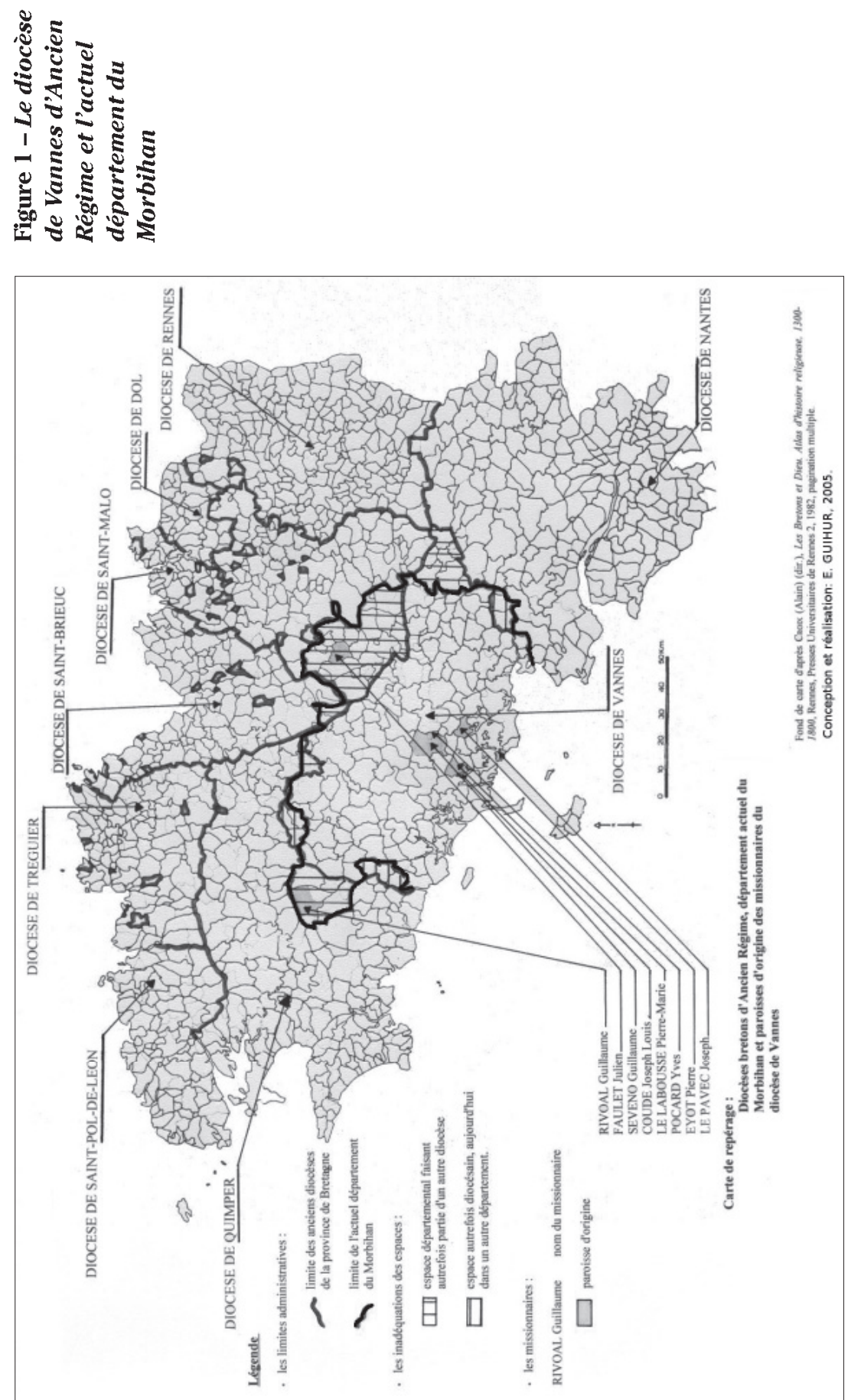
Toutefois la définition de la vocation que nous trouvons dans le Dictionnaire de Trévoux nous invite à plus d'ouverture sur les signes manifestant une vocation et sur ce qui peut la susciter. Ainsi, deux sortes de " vocation " nous sont proposées : "L'une extérieure, et l'autre intérieure. La vocation extérieure est celle qui consiste dans une simple et nue proposition d'objets, et la vocation intérieure est celle qui rend la première efficace par les secours intérieurs qui disposent nos facultés à recevoir les objets ${ }^{4}$."

Il faut donc considérer avec un grand intérêt cette " proposition d'objets ". Cette dernière invite à considérer les types de microsociétés qu'analyse Yannick Essertel dans un article sur les réseaux et les vocations missionnaires ${ }^{5}$. Ces microsociétés ou réseaux que sont la famille, la paroisse, les écoles congrégationistes, les petits et grands séminaires, constituent des milieux favorables à l'émergence d'une vocation. Les deux dernières institutions sont difficilement transposables pour la période 1660-1790 : la première parce que l'organisation scolaire de l'Ancien Régime est très différente de celle de la période étudiée par Yannick Essertel, notamment parce que le nombre d'écoles est peu élevé; la seconde, parce que le terme de petit séminaire ne semble pas avoir été utilisé dans la province de Bretagne. Certes l'enseignement reçu au collège entre dans la formation des prêtres mais il ne semble pas y avoir de distinction entre enseignement secondaire et petit séminaire : il n'existe pas deux établissements distincts ${ }^{6}$. En revanche, la famille et la paroisse sont deux types de réseaux ré-exploitables avec quelques aménagements. Ainsi la famille en Bretagne d'Ancien Régime est connue selon un mode plus horizontal que vertical ${ }^{7}$.

Le diocèse de Vannes sous l'Ancien Régime est différent de l'actuel diocèse de Vannes qui reprend les limites du département du Morbihan, au nord et à l'est, principalement. Deux missionnaires, Guillaume Rivoal et Julien Faulet, présentés comme originaires du Morbihan par les listes actuelles de la Société des Missions Étrangères de Paris ${ }^{8}$, sont à exclure de ce diocèse vannetais.

4. Dictionnaire universel françois et latin, vulgairement appelé Dictionnaire de Trévoux..., op. cit., p. 447.

5. ESSERTEL, Yannick, « Réseaux et vocations missionnaires dans le diocèse de Lyon de 1815 à 1962 », dans Revue d'Histoire Ecclésiastique, Louvain, 1995, vol 90, n 1-2, p. 69.

6. On peut toutefois noter que dans ces collèges, les élèves dévots étaient regroupés en confrérie, d'après les témoignages sur les collèges jésuites, et créaient une société secrète. Si le collège de Vannes en a eu, ce qui est probable, les archives n'ont pas de documents à ce sujet.

7. SEGALEN, Martine, Quinze générations de Bas-Bretons, parenté et société dans le pays bigouden Sud, 1720-1980, Paris, Presses Universitaires de France, 1985, 405 p.

8. Moussay, Gérard, Appavou, Brigitte, État de la Société des Missions Étrangères 16581998, Paris, Églises d'Asie, 2 vol., ordre chronologique et ordre alphabétique, 129 p. et $129 \mathrm{p}$. 
Tableau 1 - Les missionnaires vannetais de la Société des Missions Étrangères de Paris et le département du Morbihan (1660-1791)

\begin{tabular}{|l|l|l|l|l|l|l|c|}
\hline \multicolumn{1}{|c|}{ Nom } & \multicolumn{1}{|c|}{ Prénom } & Naissance & Paroisse & Ordination & Départ & Mission $\left(1^{\text {re }}\right)$ & Décès \\
\hline Rivoal & Guillaume & $31 / 12 / 1706$ & Gourin & $1 / 01 / 1733$ & $10 / 02 / 1734$ & Cochinchine & $29 / 11 / 1769$ \\
\hline Coudé & $\begin{array}{l}\text { Joseph } \\
\text { Louis }\end{array}$ & $4 / 10 / 1750$ & $\begin{array}{l}\text { Saint- } \\
\text { Gildas } \\
\text { (Auray) }\end{array}$ & $17 / 12 / 1773$ & $29 / 11 / 1773$ & Siam & $8 / 01 / 1785$ \\
\hline Faulet & Julien & $21 / 11 / 1741$ & Guilliers & $4 / 03 / 1768$ & $29 / 11 / 1773$ & Cochinchine & $31 / 12 / 1783$ \\
\hline Seveno & Guillaume & $1 / 01 / 1748$ & $\begin{array}{l}\text { Grand- } \\
\text { Champ }\end{array}$ & $19 / 09 / 1772$ & $21 / 12 / 1785$ & Pondichéry & $5 / 02 / 1788$ \\
\hline Eyot & Pierre & $28 / 04 / 1762$ & $\begin{array}{l}\text { Saint- } \\
\text { Patern } \\
\text { (Vannes) }\end{array}$ & $31 / 12 / 1786$ & $20 / 01 / 1787$ & Tonkin Occ. & $29 / 07 / 1827$ \\
\hline Pocard & Yves & $14 / 02 / 1761$ & Locmaria & $23 / 09 / 1786$ & $20 / 09 / 1787$ & Cochinchine & $14 / 02 / 1790$ \\
\hline $\begin{array}{l}\text { Le } \\
\text { Labousse }\end{array}$ & Pierre Marie & $22 / 01 / 1760$ & Pluneret & $18 / 09 / 1784$ & $20 / 09 / 1787$ & Cochinchine & $25 / 04 / 1801$ \\
\hline Le Pavec & Joseph & $21 / 08 / 1758$ & Arzon & $22 / 09 / 1787$ & $4 / 12 / 1788$ & Tonkin Occ. & $22 / 06 / 1814$ \\
\hline
\end{tabular}

\section{Des réseaux familiaux?}

Les auteurs ayant travaillé sur le recrutement sacerdotal soulignent le rôle de la famille dans la naissance de la vocation religieuse. L'existence d'un ancêtre ou d'un parent religieux contemporain du missionnaire ainsi que de fondateurs de congrégations dans la famille serait un élément favorable à l'émergence d'une vocation " traditionnelle ", locale ou missionnaire. Des documents de la main de la personne concernée ou des propos rapportés ainsi que l'utilisation de la méthode prosopographique peuvent appuyer une telle hypothèse.

Dans le cas des missionnaires vannetais ${ }^{9}$ de la Société des Missions Étrangères de Paris, il n'existe pas de lettres expliquant leur vocation ou le choix de leur départ ${ }^{10}$. Pourtant, se présenter Rue du Bac puis s'engager aux Missions Étrangères de Paris constitue, de fait, une démarche pour partir en Asie, puisque la Société ne recrute que des hommes destinés à ces régions. La correspondance (ultérieure) de ces hommes n'évoque pas les raisons de leur choix. Une fois arrivés en mission, ces hommes écrivent principalement à leurs directeurs, et essentiellement pour donner des informations d'ordre administratif.

Il ne semble pas que les missionnaires vannetais aient un ancêtre missionnaire ni un parent religieux. Aucun missionnaire n'a de frères ou de sœurs missionnaires ni même religieux. Les frères et sœurs fondent des familles en restant dans la paroisse d'origine ou dans les paroisses avoisinantes.

9. Nous utilisons l'adjectif de "vannetais " pour tous les missionnaires originaires du diocèse de Vannes, et non pour les seuls originaires de la ville de Vannes.

10. Nous ne considérons que la période concernée. Des lettres de candidature apparaissent au cours du XIX ${ }^{\mathrm{e}}$ siècle dans cette société. 
Ces missionnaires viennent de paroisses parfois éloignées de plusieurs lieues. Ces paroisses ne semblent pas avoir entre elles d'échange de curés, de prêtres, ni de mariage entre paroissiens. Il ne semble pas y avoir de lien entre les paroisses d'origine et d'autres missionnaires déjà en Asie lors de la jeunesse du futur missionnaire vannetais ni même entre les paroisses d'origine et d'autres organisations catholiques missionnaires à destination de l'Asie. Peut-être faudrait-il ouvrir aux autres destinations? Dans ce cas, l'hypothèse d'une relation privilégiée entre l'Asie et la province de Bretagne, particulièrement du diocèse de Vannes, ne serait plus valide. Or une telle relation existe bien dans les domaines des matériaux et de la culture ${ }^{11}$. Les paroisses des hommes qui nous intéressent, à savoir : Auray, Arzon, Grand-Champ, Locmaria, Pluneret et Vannes, ne sont pas missionnées pendant la période de vie de ces hommes. Les futurs missionnaires ne peuvent donc pas avoir rencontré un missionnaire en action dans leur paroisse.

Il est difficile de parler de familles donnant de nombreux enfants à l'Église comme c'est le cas au XIX ${ }^{\mathrm{e}}$ siècle. Dans l'une des familles, les Le Labousse, nous trouvons, au dix-neuvième siècle, un autre missionnaire. Il n'ira pas en Asie mais en Afrique. L'exemple de son ancêtre a-t-il compté dans le choix de la vie missionnaire? Il est difficile d'en juger. Après le départ, les familles n'ont eu que peu de nouvelles. Le départ est une rupture, d'une certaine façon une mort pour la famille. La vocation missionnaire d'Ancien Régime semble, ici, plus être le fait d'un individu que d'une famille, voire d'une poursuite de l'histoire familiale. Faut-il comparer cet engagement à celui des mousses et des marins? Pour recruter l'ensemble du personnel dont le navire a besoin, les capitaines doivent faire appel aux milieux ruraux sans lien avec le monde de la mer. Les familles ne sont pas organisées pour envoyer un des leurs en mer, surtout dans des voyages au long cours. L'engagement est plutôt une nécessité économique qu'une tradition familiale.

Faut-il pour autant exclure aussi vite la famille? Si l'on s'intéresse aux données matérielles, les seules que l'on retrouve systématiquement dans les archives, nous pouvons peut-être constater des types de famille de missionnaires.

\section{Le missionnaire, la “dîme ecclésiastique »?}

D'après les travaux de François de Dainville ${ }^{12}$, les candidats au noviciat toulousain de la Compagnie de Jésus entre 1571 et 1602 appartenaient

11. Sur ces thèmes, nous renvoyons à l'ensemble des travaux de maîtrise, DEA, thèse puis de Master I et II et doctorat en histoire moderne et contemporaine des Universités de Bretagne Sud et de Nantes, dirigés par les professeurs Jacques Weber et Gérard Le Bouëdec.

12. DAINVILLE, François de, « Le Recrutement du noviciat toulousain des jésuites de 1571 à 1586 ", dans DAINVILLE, François de, L'Éducation des jésuites XVe-XVIII siècles, Paris, éditions de Minuit, 1991, p. 74-80. 
pour un tiers à des familles de un à trois enfants, un autre tiers à des familles de quatre à cinq enfants et un dernier tiers à des familles de six enfants et plus, payant ainsi une sorte de "dîme ecclésiastique " pour reprendre son expression. Il en va différemment des familles de missionnaires ${ }^{13}$.

Tableau 2 - Le missionnaire dans sa fratrie

\begin{tabular}{|c|c|c|c|}
\hline Nom du missionnaire & $\begin{array}{c}\text { Nombre d'années de } \\
\text { mariage des parents } \\
\text { lors de la naissance }\end{array}$ & $\begin{array}{c}\text { Nombre d'enfants/ } \\
\text { nombre de garçons }\end{array}$ & $\begin{array}{c}\text { Rang de naissance } \\
\text { dans la fratrie totale/ } \\
\text { pour les garçons }\end{array}$ \\
\hline Joseph Louis Coudé & 3 & $9 / 5$ & $3 / 1$ \\
\hline Pierre Eyot & 2 & $3 / 3$ & $1 / 1$ \\
\hline Yves Pocard & 19 & $4 / 2$ & $4 / 2$ \\
\hline $\begin{array}{c}\text { Pierre-Marie Le } \\
\text { Labousse }\end{array}$ & 21 & $4 / 3$ & $4 / 3$ \\
\hline Joseph Le Pavec & 2 & $2 / 1$ & $1 / 1$ \\
\hline
\end{tabular}

Il est difficile de comparer ces comportements avec ceux des familles des séminaristes diocésains puisque l'auteur de l'étude sur le recrutement des prêtres du diocèse de Vannes n'a pas étudié précisément le rang des naissances. Il affirme toutefois que " le recrutement des clercs est lui-même très lié aux milieux aisés de la société qu'ils soient paysans, bourgeois ou nobles pour lesquels ils représentent une orientation pour les puînés de la famille ${ }^{14}$ ». Ces caractéristiques ne se retrouvent pas systématiquement chez les missionnaires. Ceux-ci sont issus soit d'une famille de cinq à six personnes, taille classique pour la période d'Ancien Régime, et sont soit des enfants tardifs, derniers-nés de la fratrie et derniers-nés des garçons (Pierre-Marie Le Labousse, Yves Pocard); soit d'une famille de taille plus restreinte, trois enfants, ou plus nombreuse, neuf enfants, et sont soit des enfants nés rapidement, soit un des premiers nés, soit le premier-né des garçons (Joseph Louis Coudé, Pierre Eyot, Joseph Le Pavec). Deux types de situation donc : une classique dans le cas d'un garçon tardif, une plus surprenante dans le cas d'un premier-né.

Le recrutement missionnaire se distingue-t-il de façon plus marquée du recrutement diocésain si l'on considère les familles sous l'angle économique? Les indices du niveau économique, que nous avons pu relever, sont le métier du père et le niveau de la capitation. Cette dernière information est à considérer à l'échelle du diocèse et à celle de la paroisse.

13. JEAN, Claire-Marie, Les Vocations nantaises de la Société des Missions Étrangères de Paris aux XIX $X^{e}$ et XX $X^{e}$ siècles, Paris, Église d'Asie, p. 106.

14. MoRHAN, Alain, Étude de démographie sacerdotale. Recrutement, origine géographique et formation des prêtres du diocèse de Vannes au XVIIf siècle, mémoire de maîtrise, sous la direction de Christian Hermann, Université de Nantes, 1993, p. 132. 


\section{Les missionnaires, des prêtres différents?}

Dans son ouvrage sur Vannes, l'historien Timothy Le Goff a cherché à situer le clergé séculier et régulier du Vannetais dans la société rurale ${ }^{15}$. Il met en évidence des tendances sociales et économiques dans le recrutement sacerdotal, tendances qui s'approfondissent à partir de 1750. Timothy Le Goff évoque particulièrement les décennies 1770-1780, un temps de redressement du recrutement qui atteint les niveaux de "l' "âge d'or" du $\mathrm{XVII}^{\mathrm{e}}$ siècle ${ }^{16}$ ". Les caractéristiques majoritaires de ce recrutement tardif sont les suivantes ${ }^{17}$ : l'origine diocésaine (locale), l'appartenance à la paysannerie aisée, la croissance du nombre de ruraux dans les postes importants du bas-clergé diocésain.

Les situations des missionnaires correspondent-elles aux caractéristiques des situations des prêtres diocésains?

\section{Tableau 3 - Familles de missionnaires, comportement familial} et niveau social

\begin{tabular}{|l|c|c|c|c|c|}
\hline $\begin{array}{l}\text { Nom du } \\
\text { missionnaire }\end{array}$ & $\begin{array}{c}\text { Nombre } \\
\text { d'années } \\
\text { mariage/ } \\
\text { naissance }\end{array}$ & $\begin{array}{c}\text { Nombre } \\
\text { d'enfants/ } \\
\text { nombre de } \\
\text { garçons }\end{array}$ & $\begin{array}{c}\text { Rang de } \\
\text { naissance } \\
\text { dans la fratrie } \\
\text { totale/ pour } \\
\text { les garçons. }\end{array}$ & $\begin{array}{c}\text { Métier } \\
\text { Année } \\
\text { d'ordination }\end{array}$ & $\begin{array}{c}\text { du père/ } \\
\text { capitation } \\
\text { année du } \\
\text { sous-diaconat }\end{array}$ \\
\hline $\begin{array}{l}\text { Joseph Louis } \\
\text { Coudé }\end{array}$ & 3 & $9 / 5$ & $3 / 1$ & $17 / 12 / 1773$ & Négociant / 40 \\
\hline Pierre Eyot & 2 & $3 / 3$ & $1 / 1$ & $31 / 12 / 1786$ & Chapelier / 3 \\
\hline Yves Pocard & 19 & $4 / 2$ & $4 / 2$ & $23 / 09 / 1786$ & Laboureur / 5 \\
\hline $\begin{array}{l}\text { Pierre-Marie } \\
\text { Le Labousse }\end{array}$ & 21 & $4 / 3$ & $4 / 3$ & $18 / 09 / 1784$ & $\begin{array}{c}\text { Laboureur } \\
/ 24\end{array}$ \\
\hline $\begin{array}{l}\text { Joseph Le } \\
\text { Pavec }\end{array}$ & 2 & $2 / 1$ & $1 / 1$ & $22 / 09 / 1787$ & Matelot $/ 3$ \\
\hline
\end{tabular}

Deux types de situation peuvent être observés : soit une famille aux revenus relativement élevés comptant parmi les plus grosses capitations des paroisses de résidence des parents (Joseph Louis Coudé et PierreMarie Le Labousse, Yves Pocard); soit une famille aux revenus modestes qui a des difficultés à constituer la rente du titre clérical de leur enfant (Pierre Eyot, Joseph Le Pavec).

La situation de Pierre-Marie Le Labousse s'inscrit dans ce que décrit Timothy Le Goff. Cela est moins vrai pour les autres missionnaires. Ces prêtres appartiennent pourtant aux catégories représentées chez les candidats à l'ordination, sans en respecter les proportions. Y a-t-il relation entre désir de mission et condition économique des parents? Cette relation doit être à présent envisagée de façon semblable à ce qu'écrit François de Dainville

15. LE Goff, Timothy, Vannes et sa région, ville et campagne dans la France du XVIIr siècle, Loudéac, Yves Salmon, 1989, p. 225-260.

16. Ibidem, p. 227.

17. Ibid., p. 229. 
à propos des écoliers du collège de Troyes : en temps normal, les cours du blé influent sur l'effectif scolaire ${ }^{18}$.

\section{À la croisée des données : \\ famille et situation économique et comportement diocésain}

Auparavant, intéressons-nous aux deux critères que sont la place du missionnaire dans sa fratrie et le niveau économique de sa famille.

Tableau 4 - Une spécificité des familles de missionnaires?

\begin{tabular}{|c|c|c|c|c|}
\hline & \multicolumn{2}{|c|}{ Critère familial } & \multicolumn{2}{c|}{ Critère économique } \\
\cline { 2 - 5 } $\begin{array}{c}\text { Nom du } \\
\text { missionnaire }\end{array}$ & $\begin{array}{c}\text { Famille classique, } \\
\text { enfant tardif et } \\
\text { dans les derniers- } \\
\text { nés }\end{array}$ & $\begin{array}{c}\text { Famille de taille } \\
\text { remarquable, } \\
\text { premier né }\end{array}$ & $\begin{array}{c}\text { Famille aux } \\
\text { revenus élevés }\end{array}$ & $\begin{array}{c}\text { Familles aux } \\
\text { revenus faibles }\end{array}$ \\
\hline $\begin{array}{c}\text { Joseph Louis } \\
\text { Coudé }\end{array}$ & $\mathrm{X}$ & $\mathrm{X}$ & $\mathrm{X}$ & \\
\hline $\begin{array}{c}\text { Pierre-Marie Le } \\
\text { Labousse }\end{array}$ & $\mathrm{X}$ & $\mathrm{X}$ & $\mathrm{X}$ & \\
\hline Yves Pocard & & $\mathrm{X}$ & & $\mathrm{X}$ \\
\hline Pierre Eyot & & $\mathrm{X}$ & $\mathrm{X}$ \\
\hline Joseph Le Pavec & & & & \\
\hline
\end{tabular}

Si l'on met à part la famille Coudé qui participe au négoce avec l'Asie, dont le père fournit les navires de la Compagnie des Indes en blé et dont le fils part en Asie avant d'être ordonné prêtre, deux types de cas se dégagent : ou bien le missionnaire est un enfant tardif, parmi les derniers-nés des garçons de la fratrie, appartenant à une famille relativement aisé ou bien le missionnaire est un des premiers enfants, parfois le premier garçon d'une famille aux revenus plutôt faibles. Les familles des missionnaires ne font pas partie de la catégorie la plus pourvoyeuse de l'Église, mais elles correspondent pour partie aux caractéristiques du recrutement diocésain. Elles ne présentent toutefois pas les caractéristiques les plus répandues de ces familles sources. C'est pourquoi il faut sans doute considérer l'engagement personnel de l'enfant ainsi que la situation économique générale.

D’après le tableau présenté par Timothy Le Goff, le Vannetais connaît une période de prospérité économique entre 1780 et 1784 . Or, la date de tonsure puis celle de l'établissement du titre clérical - les missionnaires s'engagent ainsi dans la voie de la prêtrise -, se situent dans la période prospère, 1780-1784, à l'exception de Joseph Louis Coudé, fils d'un négociant en contact avec la Compagnie des Indes, et de Joseph Le Pavec qui est âgé lorsqu'il commence ce cursus ${ }^{19}$. En revanche, le départ correspond,

18. DAINVILLE, François de, "Effectifs des collèges et scolarité aux dix-septième et dixhuitième siècles dans le nord-est de la France ", dans DaInviLle, François de, L'Éducation des jésuites, op. cit., p. 91.

19. Joseph Le Pavec n'apparaît sur les affiches du collège de Vannes qu'en 1784, il aurait alors 26 ans et serait en philosophie. 
pour tous les missionnaires, à la période de crise que traverse la région entre 1785 et 1789 . Au même moment, le nombre de candidats aux cures est plus élevé que le nombre de cures vacantes. Dans ce cas les familles n'ont pas toujours la possibilité de subvenir aux besoins de ce fils sans s'endetter (les familles l'ont déjà fait pour les titres cléricaux). Partir serait-il une solution à un problème économique? Cependant, la solution à ce problème financier est limitée puisque la Société des Missions Étrangères de Paris souhaite bénéficier des revenus de ce titre.

Le cadre paroissial serait-il plus adapté pour cette étude? Faut-il replacer le recrutement missionnaire dans le cadre du recrutement clérical de la paroisse? En d'autres termes, les missionnaires viennent-ils des paroisses les plus fécondes en prêtres?

Tableau 5 - Missionnaires et recrutement paroissial

\begin{tabular}{|c|c|c|}
\hline Nom du missionnaire & Paroisse d'origine & $\begin{array}{c}\text { Nombre de prêtres fournis } \\
1780-1790^{\text {a }}\end{array}$ \\
\hline Joseph Louis Coudé & Auray & $1-2$ \\
\hline Pierre-Marie Le Labousse & Pluneret & $3-4$ \\
\hline Yves Pocard & Grandchamp & $7-8$ \\
\hline Pierre Eyot & Vannes & 14 et + \\
\hline Joseph Le Pavec & Arzon & $1-2$ \\
\hline
\end{tabular}

a. MoRHAN, Alain, Étude de démographie sacerdotale, op. cit., p. 78.

Les missionnaires viennent de paroisses dont le comportement est fort différent. Encore faudrait-il mesurer la proportion des jeunes gens qui deviennent prêtres par tranche d'âge afin d'affiner l'étude. Les missionnaires vannetais de la Société des Missions Étrangères sont plutôt originaires de l'intérieur et correspondent en cela au recrutement diocésain. Le niveau financier des familles est plutôt hors moyenne, nettement au-dessous et tout aussi nettement au-dessus. Enfin, les origines géographiques ne respectent pas les tendances de recrutement général du diocèse. La paroisse peut-elle tout de même entraîner le choix préférentiel d'une destination asiatique? Nous venons de voir les raisons des choix d'une mission en Asie des jésuites français au XVII ${ }^{\mathrm{e}}$ siècle ${ }^{20}$. Dans le cas particulier de Vannes nous pouvons nous demander si la proximité au port de Lorient et celui de la Compagnie des Indes est un facteur de choix de l'Asie.

\section{Des réseaux paroissiaux?}

Le second critère qu'envisage Yannick Essertel est le réseau paroissial, avec en figure de proue quelques personnalités remarquables ${ }^{21}$.

20. Voir la contribution d'Amélie Vantard dans le même volume.

21. ESSERTEL, Yannick, "Réseaux et vocations missionnaires dans le diocèse de Lyon de 1815 à 1962 ", art. cité, p. 53. 


\section{La paroisse, premier lieu d'expression de la dévotion}

Il semble à Yannick Essertel que la paroisse soit un relais de la famille par le biais d'associations pieuses. L'association pieuse prend, au XVII comme au $\mathrm{XVIII}^{\mathrm{e}}$ siècle, la forme de la confrérie de dévotion. Ces confréries sont beaucoup plus nombreuses et actives au XVII ${ }^{\mathrm{e}}$ qu'au XVIII ${ }^{\mathrm{e}}$ siècle mais il n'en reste plus de traces. L'auteur du travail sur les confréries dans le diocèse de Vannes ${ }^{22}$ fait état de l'indigence des sources, en particulier de listes de confrères qui font cruellement défaut pour connaître un peu plus le public des confréries. Il semble d'ailleurs ne pas exister de documentation sur les confréries des paroisses d'origine des missionnaires qui nous intéressent ici. D'après la carte de répartition des confréries ${ }^{23}$, les paroisses d'origine des missionnaires sont très diversement concernées par ce phénomène.

Pour estimer le degré de religiosité, nous ne pouvons utiliser que le degré de participation de la paroisse dans le recrutement sacerdotal diocésain. Le comportement des candidats aux missions ne semble pas correspondre totalement aux caractéristiques du recrutement des candidats à la prêtrise. Faut-il y voir un engagement pour l'Asie plus qu'un engagement missionnaire? En effet nous ne pouvons, faute de matériaux, déterminer les caractéristiques de la vocation missionnaire en Asie des membres de la Société des Missions Étrangères de Paris.

\section{La paroisse et l'Asie}

Des travaux ont été réalisés par Timothy Le Goff sur le recrutement des officiers embarqués à bord des navires de la Compagnie des Indes ${ }^{24}$. La cartographie du recrutement met en avant le rôle majeur de deux ports pour la Bretagne dans les relations au lointain, Nantes et Lorient. Il semble que dans la culture lorientaise, la présence de l'Asie soit durablement installée : «On [y] parle de Pondichéry comme de n’importe quelle ville du royaume ${ }^{25}$. " Le recrutement (lieu de domicile) des marins se fait dans un territoire assez proche de Lorient qui correspond à la zone de recrutement des missionnaires. Si l'on considère également les lieux d'origine des officiers et matelots classés, donc susceptibles de s'embarquer sur les navires de la nouvelle Compagnie en 1785, nous nous apercevons que les paroisses

22. LE GAC, Jean-Luc, Les Confréries de dévotion dans le diocèse de Vannes aux XVIf et XVII siècles, mémoire de maîtrise sous la direction de Jean Quéniart, Université de Rennes 2- Haute Bretagne, 1980, 116 p.

23. Ibidem, p. 45.

24. LE Goff, Timothy, "Le Recrutement géographique et social des gens de mer bretons à la fin de l'Ancien Régime (résultats préliminaires), dans TANGUY, Daniel, RouDAUT, François (dir.), La Bretagne, une province à l'aube de la Révolution. Colloque tenu à Brest les 28-30 septembre 1988, Brest - Quimper, Centre de recherche bretonne et celtique - Société archéologique du Finistère, 1989, p. 207-223.

25. LE BOUËDEC, Gérard, " La Culture Océan Indien dans le pays de Lorient au XVIII siècle ", dans WEBER, Jacques (dir.), Les Relations entre la France et l'Inde de 1673 à nos jours, Paris, Les Indes Savantes, 2002, p. 223. 
d'origine des missionnaires se situent dans cette zone de recrutement (voir en annexe : Missionnaires et recrutement des marins du port de Lorient, 1768 et 1785$)$.

Il reste à rechercher des indices de présence matérielle ou imaginaire de l'Asie dans ces paroisses et dans les familles des missionnaires, avant le départ des leurs. Une autre hypothèse est à considérer, celle de la pluriactivité religieuse : faut-il interpréter le départ en mission comme une activité de substitution pour un clerc? La mer offrirait alors une autre activité de la même façon qu'elle l'est pour des populations littorales tournées naturellement vers le monde rural et ses métiers. Il nous faut donc nous intéresser aux populations religieuses des navires à destination de l'Asie.

Le monde religieux est représenté sur les navires de la Compagnie des Indes par les aumôniers. La vocation de l'aumônier est différente de celle du missionnaire : si tous les deux embarquent et s'intéressent aux âmes des marins, l'aumônier revient dans son pays d'origine, le missionnaire est envoyé sans espoir de retour. Tous deux voyagent sur les navires de la Compagnie des Indes à destination de l'Asie dans des conditions plutôt différentes, l'un intégré à l'équipe des officiers, l'autre aux passagers. Ces aumôniers ont été étudiés par Philippe Haudrère ainsi que par Philippe Zérathe dans leurs thèses sur la Compagnie des Indes ${ }^{26}$ et sur les gens de mer du diocèse de Vannes ${ }^{27}$.

Tableau 6 - Origine diocésaine des aumôniers de la Compagnie des Indes et des missionnaires de la Société des Missions Étrangères de Paris

\begin{tabular}{|l|c|c|c|}
\hline \multirow{2}{*}{ Généralité de } & $\begin{array}{c}\text { Origine des aumôniers } \\
(\%)^{a}\end{array}$ & France & Brigine des missionnaires (\%) \\
\cline { 3 - 4 } & 0 & 0 & 0 \\
\hline Dol & 5,6 & 1,74 & 14,3 \\
\hline Nantes & 21,6 & 1,3 & 11,4 \\
\hline Quimper & 8,4 & 4,17 & 34,3 \\
\hline Rennes & 3,7 & 0,35 & 2,9 \\
\hline Saint-Brieuc & 19,8 & 0,35 & 2,9 \\
\hline Saint-Malo & 6,6 & 1,04 & 8,6 \\
\hline Saint-Pol-de-Léon & 0,9 & 1,04 & 8,6 \\
\hline Tréguier & 33 & 2,08 & 17,1 \\
\hline Vannes & & & \\
\hline
\end{tabular}

a. Chiffres extraits de HAUDRÈre, Philippe, op. cit., p. 423.

D'après les données présentées dans le tableau 6, il semble qu'à l'exception de Rennes, plus la généralité est proche de Lorient et plus elle est

26. HAUDRÈRE, Philippe, La Compagnie française des Indes au XVIIe siècle, Paris, Librairie de l'Inde, 1989, 1429 p. [rééd. Paris, les Indes Savantes, 2005, 1087 p.].

27. ZERATHE, Philippe, Les Gens de mer du département maritime de Vannes (fin XVIe-fin XVIIf siècles) : métiers, statuts, identités, thèse de troisième cycle, sous la direction de Alain Cabantous, Université Paris I - Panthéon-Sorbonne, 2000, 565 p. 
impliquée dans le commerce asiatique (Saint-Malo étant à part), plus il y a de vocations missionnaires dans cet espace.

Les aumôniers sont majoritairement des séculiers (61,3 \%). Les autres appartiennent aux grandes familles religieuses: Augustins, Carmes, Dominicains et Franciscains. Ces hommes ont plus de 26 ans à leur premier voyage. Ce n'est pas le cas des missionnaires des Missions Étrangères de Paris. Est-ce dû à la formation ou à la destination de la société ou congrégation? Les membres des Missions Étrangères de Paris n'ont pas d'autre vocation que d'être missionnaires en Asie. Les ordres religieux déjà cités ne fournissent pas que des aumôniers et, dans le cas de missionnaires, ceuxci ne se destinent pas exclusivement à l'Asie. Il ne faut pas oublier que les Missions Étrangères de Paris peinent à trouver des candidats durant cette période. Est-ce pour cela que ces hommes partent jeunes? Ce serait alors contraire aux conseils des Instructions ${ }^{28}$ qui prônent la recherche et l'envoi de missionnaires aguerris.

Tableau 7 - Âge à l'embarquement des missionnaires vannetais

\begin{tabular}{|l|c|c|c|}
\hline Missionnaire & Date de naissance & Date d'embarquement & $\begin{array}{c}\text { Age à } \\
\text { l'embarquement }\end{array}$ \\
\hline Joseph Louis Coudé & $4 / 10 / 1750$ & $29 / 11 / 1773$ & 23 \\
\hline Pierre-Marie Le Labousse & $22 / 01 / 1760$ & $20 / 09 / 1787$ & 27 \\
\hline Yves Pocard & $14 / 02 / 1761$ & $28 / 09 / 1787$ & 26 \\
\hline Pierre Eyot & $28 / 04 / 1762$ & $20 / 08 / 1787$ & 25 \\
\hline Joseph Le Pavec & $21 / 08 / 1758$ & $4 / 12 / 1788$ & 30 \\
\hline
\end{tabular}

Rien de comparable donc aux réseaux que dessine Yannick Essertel dans les paroisses du diocèse de Lyon. Le rôle du prêtre de la paroisse, l'influence qu'il peut avoir sur ses ouailles n'a pas laissé de traces suffisantes pour déterminer son influence dans l'éclosion de vocations missionnaires. Encore faut-il que cette influence ait été réelle. Il ne semble pas que des exemples de départs missionnaires originaires de la paroisse aient eu un effet d'entraînement. Nous ne trouvons, ni pour le $\mathrm{XVII}^{\mathrm{e}}$ ni pour le $\mathrm{XVIII}^{\mathrm{e}}$ siècle, d'engagement dans la Société de prêtres originaires de la même paroisse. L'auteur de l'étude sur les vocations lyonnaises propose une troisième microsociété favorable à l'éclosion d'une vocation missionnaire : les réseaux des congrégations ou écoles. Yannick Essertel écrit que la formation scolaire, plus exactement la rencontre avec un personnage du collège, est déterminante dans l'éclosion et la réalisation d'une vocation missionnaire ${ }^{29}$.

28. Les Instructions sont rédigées par deux des fondateurs de la Société, Mgr Pallu et Lambert de la Motte. Elles visent à guider les membres de la Société restés à Paris dans le recrutement des candidats aux missions. Ces instructions servent également à guider les missionnaires sur place, comment régler leur conduite, etc.

29. ESSERTEL, Yannick, "Réseaux et vocations missionnaires dans le diocèse de Lyon de 1815 à 1962 ", art. cité, p. 63. 


\section{Lieu d'enseignement : lieu du désir de mission?}

Nous limiterons notre étude au collège de Vannes. Il n'y a, en effet, que cet établissement qui donne l'éducation nécessaire à l'entrée au séminaire dans le diocèse. Nous ne nous intéresserons pas au séminaire par manque relatif d'information à ce propos. Les documents sont de nature administrative sans possibilité de déterminer l'influence de l'enseignement dans le désir de mission ${ }^{30}$. D'autre part une partie des missionnaires a étudié à Paris et y a probablement fait son séminaire puisque l'on retrouve des dimissoires dans les insinuations ecclésiastiques, notamment pour Pierre Eyot ${ }^{31}$.

\section{La fréquentation de l'exotisme dans le collège de Vannes}

Nous ne trouvons pas de produits exotiques dans les maisons des familles de missionnaires (dans les inventaires après décès que nous avons étudiés ${ }^{32}$ ). Les travaux réalisés sur la diffusion des objets exotiques (tissus et sucre) ont montré que les familles sont en contact avec cet exotisme plus rapidement en ville qu'en campagne et plus rapidement en haut de l'échelle sociale qu'en bas. Cela semble s'appliquer aux familles des missionnaires. Elles ne semblent pas avoir de lien matériel direct avec l'Asie, toujours à l'exception notable de la famille de Joseph Louis Coudé. Joseph Coudé (le père) fait du commerce de grains avec la Compagnie des Indes. Nous pouvons donc supposer qu'il possède quelques objets asiatiques. Il faut également noter que son fils, Joseph Louis, part très jeune, à l'âge de 23 ans. Il part plus jeune que la moyenne des missionnaires vannetais (moyenne de 29 ans, médiane de 26 ans) et que la moyenne des départs de la Société. Il n'est pas encore prêtre et c'est un des rares missionnaires des Missions Étrangères de Paris dans ce cas ${ }^{33}$. Nous pouvons supposer que sa connaissance de la Compagnie et ses relations dans le milieu maritime ont favorisé cet engagement précoce.

Les Jésuites étaient les principaux et les plus prolixes agents diffuseurs d'ouvrages sur l'Asie. Leurs Relations invitaient les esprits à imaginer l'Asie : ils racontaient l'Asie. Les collèges, principalement jésuites, possédaient quelques ouvrages de ce type dans leurs bibliothèques. Après l'expulsion et la dissolution de la Compagnie de Jésus, les collèges bretons fonctionnent encore sur le modèle jésuite. Les enseignants choisis par la ville utilisent

30. Arch. dép. du Morbihan, G 745 et G 746 (anciennement 85 G 1 et 85 G 2). Alain Morhan a toutefois un peu travaillé sur la formation dans le séminaire, mais il reste au niveau de la réglementation générale. Cf. dernier chapitre de son mémoire de maîtrise, MORHAN, Alain, Étude de démographie sacerdotale, op. cit., p. 113-127.

31. Arch. dép. du Morbihan, 40 G 8, dimissoire pour l'ordination sacerdotale, 3 mai 1786.

32. Arch. dép. du Morbihan, B 5821, 23 août 1768. La famille Pocard semble plutôt à l'aise sans être très fortunée : total de l'inventaire 1471 livres 7 deniers, avec les outils de travail et animaux de la ferme.

33. Il est ordonné prêtre le 17 décembre 1773, sans doute lors de son trajet jusqu'au port de Lorient. Mais nous ne le retrouvons pas dans les registres des ordinations de la période, $40 \mathrm{G} 7$. 
les mêmes lieux et les mêmes livres. Pour preuve, les affiches postérieures à 1762 qui annoncent des exercices publics sont similaires aux précédentes (nature et thème). Dans l'inventaire de 1762, la liste des ouvrages de la bibliothèque, qui compte plusieurs centaines de titres ${ }^{34}$, est la suivante : Atlas, Histoire Universelle, Parfait vicaire apostolique, Description du monde (deux exemplaires), Le missionnaire paroissial, Les lettres édifiantes, Le chrétien en solitude, Le missionnaire parfait (deux exemplaires), La vie de saint Xavier, La vie de Saint Ignace (deux exemplaires), Instructions pour les nouveaux convertis, Journal de Siam. Le notaire note aussi la présence des ouvrages suivants : Dictionnaire Encyclopédique (sept volumes), Des religions du monde, Etat de l'église de la Chine, Lettres persanes. Ce dernier ouvrage fait partie des livres prohibés. L'Église révèle encore d'autres ouvrages : trente-cinq volumes des Lettres édifiantes, une Histoire du Japon, les Relations de la Nouvelle-France.

Ces livres et objets ouvrent à la découverte de l'Asie. Les élèves ontils accès directement à la bibliothèque? Le plus souvent, les collégiens n'entrent pas dans la bibliothèque car elle est réservée aux professeurs ${ }^{35}$. Ils ont peut-être eu quelques traces de lecture dans les cours qu'ils suivaient. Les archives des enseignements ne permettent pas de l'affirmer ${ }^{36}$. En revanche, la présence dans l'église - dont les premiers et réguliers occupants sont les écoliers - de quelques ouvrages en relation avec l'Asie laisse penser qu'ils sont utilisés pour les sermons et autres prêches. Ces ouvrages incitent à la rêverie, à l'imagination du lecteur, les auteurs prenant quelques libertés avec la réalité. Le Journal de Siam que les Jésuites conservent dans leur bibliothèque est un exemple de ces récits de voyage quelque peu imaginatifs.

Vannes est sur la route qui va de Paris à Lorient et le collège est assez indiqué comme lieu de repos pour des religieux. Leur présence fut peut-être un moyen d'édification des écoliers. Peut-être dormaient-ils à Vannes. Collège et séminaire étaient, de toute façon, très liés, puisque le collège enseignait jusqu'en philosophie et en théologie. Le séminaire était un établissement annexé au collège lors de sa fondation ${ }^{37}$. Il ne prend son " indépendance " que lors de l'expulsion des jésuites. La venue ou la présence de missionnaires à Vannes était-elle l'occasion d'un prêche pour les écoliers sur ces thèmes?

Restent les lettres des missionnaires. Le XVIII ${ }^{\mathrm{e}}$ siècle accorde, de façon générale, plus d'importance au contact, au réseau de personnes qu'à l'impact littéraire ${ }^{38}$. Peut-être faut-il considérer le cas particulier des lettres

34. Arch. dép. du Morbihan, D 6, liquidation des biens des Jésuites, inventaire des biens meubles du collège, du 9 au 30 août 1762 .

35. Si les élèves y ont accès, ils n'y ont sans doute qu'un accès réduit.

36. Arch. dép. du Morbihan, G 746, cahiers de cours des professeurs.

37. Arch. dép. du Morbihan, D 56, fondation d'un séminaire.

38. DuTEIL, Jean-Pierre, "L'Asie à travers les relations de voyage à l'époque moderne ", journées d'études sur le thème "Société et Territoire ", Université de Bretagne Sud, Lorient, 4-5 mars 2005. 
de missionnaires rassemblé dans des précis? Ceux-ci sont envoyés à un public choisi. Nous avons retrouvé un précis d'une dizaine de pages dans les archives du séminaire de Vannes ${ }^{39}$. Il provient du séminaire des Missions Étrangères de Paris et date de juin 1784. Le message qui est diffusé dans l'ensemble de ses pages est la " disette d'ouvriers ", expression d'un des missionnaires. Les entrées Rue du Bac des candidats vannetais se font entre juillet 1785 et juillet 1788 pour ceux qui partent dans les années 1780, donc après l'arrivée probable de ce précis des missions.

\section{Tableau 8 - Précis des missions et engagement} des missionnaires Rue du Bac

\begin{tabular}{|l|l|}
\hline \multicolumn{1}{|c|}{ Missionnaires } & \multicolumn{1}{|c|}{ Date d'entrée aux Missions Étrangères de Paris } \\
\hline Joseph Coudé & $16 / 07 / 1772-$ père en relation avec la CIO \\
\hline Guillaume Séveno & $11 / 05 / 1778-$ part avant au Sénégal Saint-Esprit \\
\hline Précis des Missions arrivé au séminaire & Juillet/août $1784 ?$ \\
\hline Pierre Eyot & $13 / 07 / 1785$ (probablement déjà à Paris) \\
\hline Yves Pocard & $6 / 12 / 1786$ \\
\hline Pierre-Marie Le Labousse & $15 / 03 / 1787$ \\
\hline Joseph Le Pavec & $23 / 07 / 1788$ \\
\hline
\end{tabular}

Il faut noter que ce précis est assez original pour les Missions Étrangères de Paris. La Société n'avait pas pour habitude de publier des extraits de lettres ou d'ouvrages en vue de susciter des vocations et de solliciter des donateurs. Le recrutement ne se faisait pas par le biais du récit et du rêve. Il se voulait fondé sur la motivation des missionnaires, leurs qualités et leurs expériences. La Société ne va pas rechercher les candidats. Ceux-ci se présentent aux directeurs de la Société après l'avoir découverte seuls ou par le moyen de relations personnelles. Il semble d'ailleurs que certains missionnaires se soient connus avant leur départ, sans doute avant même leur entrée dans la Société.

Il faut noter enfin une certaine sensibilité à l'Asie dans le collège. Même s'il existe une différence de vingt ans entre l'inventaire de 1762 et le départ des Vannetais, nous devons constater que le réfectoire conserve « six compotiers de porcelaine "; dans l'église, les commissaires notent la présence de " chasubles de bazin ", textile asiatique; ils mentionnent aussi de nombreuses "cartes géographiques " dans les chambres des enseignants, et dans celle du procureur " une estampe chinoise et son rouleau " mais elle n'est évaluée que 10 sous; enfin dans la chambre du père préfet, les commissaires trouvent " 19 vestes de cotton [sic]".

L'Asie n'est donc pas inconnue dans le collège. Néanmoins sa connaissance reste assez limitée et l'usage de ses produits tout autant. Cette distance entre le commun et l'exceptionnel ne peut que favoriser un émerveillement et une admiration à l'endroit de cet espace.

39. Arch. dép. du Morbihan, G 750, Précis des nouvelles des Missions des Indes Orientales reçues au séminaire des Missions Étrangères de Paris au mois de juin 1784. 


\section{Un réseau de connaissances tissé au collège?}

L'auteur d'un article sur le collège de Vannes écrit que le collège est, durant tout l'Ancien Régime, très ouvert aux horizons lointains ${ }^{40}$, notamment vis-à-vis du Canada en raison du départ d'un certain nombre d'anciens élèves vers cette destination. L'origine en serait un ancien missionnaire du Canada revenu en France puis nommé directeur du collège de Vannes, en 1667. Par la suite, plusieurs grands missionnaires au Canada auraient effectué une partie de leur scolarité dans cet établissement. Malheureusement, l'auteur ne cite pas ses sources. Il renvoie bien à quelques notices et autres articles parus mais cela reste limité. Reste donc ce que les archives consultées nous livrent, à savoir la connaissance des missionnaires entre eux et le point de contact que semble constituer un certain M. Grinne.

François de Dainville s'est intéressé au recrutement du noviciat toulousain jésuite ${ }^{41}$. Il constate que l'influence des collèges s'avère primordiale afin d'expliquer la géographie du recrutement du noviciat et que la zone d'influence des établissements " explique pour une large part celle du noviciat ${ }^{42}$ ». Il n'en reste pas moins que certaines origines géographiques peuvent surprendre. Il faut alors, d'après cet auteur, " entrevoi[r] comment des relations personnelles ont pu conduire des jeunes gens de [...] contrées [éloignées] jusqu'au noviciat de Toulouse ${ }^{43}$ ".

Le réseau est de première importance dans le choix du lieu de vie. Martine Segalen ne disait pas autre chose pour expliquer la mobilité en pays bigouden sud. Jean-Pierre Duteil évoque aussi le réseau comme le facteur premier de la connaissance de l'Asie et non plus la connaissance livresque ${ }^{44}$. Ce type de réseau pourrait exister par l'intermédiaire de la Compagnie Royale des Indes mais aussi naître au sein du collège.

Les hommes qui partent en mission avec la Société sont issus de la même génération de prêtres. Ils ne sont pas nés en même temps, en revanche, ils sont ordonnés entre 1784 et 1787. Un des premiers, Pierre Eyot, est ordonné prêtre à Paris. Les autres semblent être restés à Vannes. Ils se connaissent néanmoins car M. Pocard peut écrire à propos de M. Le Pavec que « s'il [...] vient un nouveau missionnaire nommé M[onsieu]r Le Pavec, [on] [doit] lui dir[e] qu'[il] [est] son très humble serviteur et qu'[il] [s]e recommande très particulièrement à ses prières, parce qu'[il a] une grande confiance dans ses reliques ${ }^{45}$ ".

40. MARSILLE, Henri, " Le Collège de Vannes et la Nouvelle France ", dans Bulletin Mensuel de la Société Polymathique du Morbihan, juillet 1995, t. 121, p. 31-41.

41. DAINVILLE, François de, «Le Recrutement du noviciat toulousain des jésuites de 1571 à 1586 ", dans DaInVILLE, François de, L'Éducation des jésuites, op. cit., p. 74-80. Certes la différence chronologique entre les deux périodes étudiées est importante mais ces hypothèses sont reprises pour une période postérieure à notre étude, nous pouvons donc la considérer comme possible pour l'Ancien Régime.

42. Ibidem, p. 77.

43. Ibid., p. 79.

44. DuTEIL (Jean-Pierre), "L'Asie à travers les relations de voyage... ", op. cit.

45. Archives de la Société des Missions Étrangères, M. Pocard à M. Letondal, vers 1789, vol. 801 , p. 294. 
Lorsque ces lignes sont écrites, Yves Pocard a quitté le royaume de France depuis deux ans. Or au jour de son départ, Joseph Le Pavec va être ordonné prêtre et n'a pas encore quitté Vannes. Ils ont dû se rencontrer et s'apprécier auparavant. Peut-être au collège ou au séminaire? Sans doute avaient-ils également eu connaissance de leurs desseins réciproques. Il apparaît en effet qu'un possible dénominateur commun du parcours de ces hommes soit le collège. Ils écrivent à leur famille par l'intermédiaire du sous-principal du collège $\mathrm{M}$. Grinne ou directement à celui-ci ${ }^{46}$. Nous retrouvons le patronyme de Grinne dans les archives du collège dès 1762 . Il est alors homologué en tant que régent de cinquième ${ }^{47}$. Il devient professeur de philosophie avant d'être nommé sous-principal du collège en 1772. Il semble que ce soit une personnalité dans le collège. Il est en effet l'un des seuls à y rester si longtemps. Il est considéré comme un père spirituel puisque Pierre Eyot, par exemple, commence sa lettre du 3 juin 1799 par " Monsieur et très cher Père en Dieu ${ }^{48}$ ". Il raconte ensuite les persécutions des prêtres missionnaires en Cochinchine, notamment le martyre de l'un d'entre eux et rend compte de l'ensemble des territoires missionnés par les Missions Étrangères de Paris dans la péninsule indochinoise. Il ne demande pas d'" ouvriers ${ }^{49}$ ". Il donne quelques nouvelles et demande des siennes en retour. On peut penser que ce genre de lettres servait quand même à édifier les écoliers. Une trace montre qu'elles étaient au moins lues dans le milieu dévot de Vannes, sinon aux écoliers, puisque lorsqu'un enfant du pays (ici M. Le Labousse) fait appel à la générosité de ses anciens voisins, il reçoit une réponse positive. Ainsi M. Le Labousse a-t-il bien " reçu les paquets et les caisses que [M. Grinne] [a] eu la bonté de [lui] envoyer. [M. Le Labousse] [lui] en fai[ $\mathrm{t}$ ] [s] es humbles remerciements. [Et demande de remercier] pour [lui] les charitables demoiselles ${ }^{50}$ ». Il demande ensuite à M. Grinne d'être son intermédiaire pour leur porter les lettres qu'il leur a écrites.

Il semble que M. Grinne soit tenu au courant, bien longtemps après leur départ, de la situation de ses anciens élèves. Est-ce parce que ce professeur, comme ce que rapporte Yannick Essertel à propos du père Loras, avait aussi des rêves de mission et avait encouragé les futurs missionnaires à persévérer dans cette voie? Des quatre missionnaires partis en mission et passés par le collège de Vannes dans les années 1770, tous écrivent à

46. Quelques exemples de lettres par l'intermédiaire de M. Grinne : Archives de la Société des Missions Étrangères de Paris, M. Le Labousse à M. Grinne, le 21 juillet 1798, vol 746, p. 746-749; M. Eyot à M. Grinne, le 3 juin 1799, vol. 693, p. 145-152.

47. Arch. dép. du Morbihan, D 7, "Arrest de la Cour, rendu sur les conclusions de Monsieur le Procureur Général du Roi, qui homologue les nominations des sous-principaux et professeurs des Collèges de Rennes, Vannes et Quimper ", le 19 octobre 1762, et Archives départementales d'Ille-et-Vilaine, 1 Bk8.

48. Archives de la Société des Missions Étrangères, M. Eyot à M. Grinne, le 3 juin 1799, vol. 693 , p. 145.

49. Idem.

50. Archives de la Société des Missions Étrangères, M. Le Labousse à M. Grinne, le 16 juin 1792 , vol. 746 , p. 363 . 
leurs familles par le biais de cet homme. Cela ne peut être une simple coïncidence, même s'il est difficile d'en dire plus sans découverte de nouvelles archives.

Le rapprochement des cas tirés d'une prosopographie systématique permettait à Yannick Essertel de montrer que les familles, les paroisses, les écoles congrégationistes et les séminaires formaient de véritables catalyseurs de vocations missionnaires ${ }^{51}$. Il est difficile à l'échelle du diocèse de Vannes et à partir du petit nombre de missionnaires partis en Asie de valider complètement ces quatre types de microsociétés. Il semble toutefois que certaines sont plus importantes que d'autres, notamment le collège avec quelques personnages clefs et le réseau des candidats missionnaires.

Les archives disponibles ne répondent malheureusement pas, à la différence des indipetae, à la nature de la vocation missionnaire. En revanche nous pouvons dresser un certain portrait du missionnaire des Missions Étrangères de Paris dans le diocèse de Vannes. Issu d'une famille de taille plutôt moyenne, il est le dernier-né des garçons de la fratrie. À l'inverse, lorsque la famille est de taille remarquable, le missionnaire est le premier né voire le seul garçon vivant. Rien n'indique que ces familles soient particulièrement dévotes, leurs paroisses de résidence ne participant pas de la même façon au recrutement diocésain. Il ne semble pas non plus que les familles aient un rapport avec l'Asie à l'exception de la famille Coudé, dont le missionnaire se distingue par un engagement précoce, avant même l'ordination sacerdotale. Le critère économique semble en revanche pertinent. Les candidats aux missions asiatiques ${ }^{52}$ entrent aux Missions Étrangères de Paris lors de changement de conjoncture, alors que la concurrence se fait plus rude pour l'obtention d'une charge de curé, notamment dans leur paroisse d'origine. Toutefois cela ne résout pas la question du choix du continent de mission.

En revanche si l'on considère des facteurs de la vie " séculière ", il semble y avoir un certain lien de proximité avec les zones de recrutement de la Compagnie des Indes Orientales. La connaissance de l'Asie, rêvée ou réelle, est probablement en lien avec ce choix, d'autant que le collège dans lequel les futurs missionnaires étudient est marqué par une sensibilité à l'Asie.

Il résulte donc de cette rapide évocation du climat dans lequel vivent les candidats aux missions des Missions Étrangères de Paris, que la volonté d'engagement sur le terrain des missions asiatiques naît probablement de rencontres fortes, notamment au cours de la scolarité dans le collège, avec le personnage clef à Vannes de M. Grinne, professeur et sous-principal durant les décennies $1760-1770$ et 1780 .

51. ESSERTEL (Yannick), « Réseaux et vocations missionnaires dans le diocèse de Lyon de 1815 à 1962 ", art. cité, p. 66.

52. Pour valider cette hypothèse, il faudrait comparer ces engagements à l'ensemble des départs en mission du diocèse. 
Annexe - Missionnaires et recrutement des marins du port de Lorient (1768 et 1785)

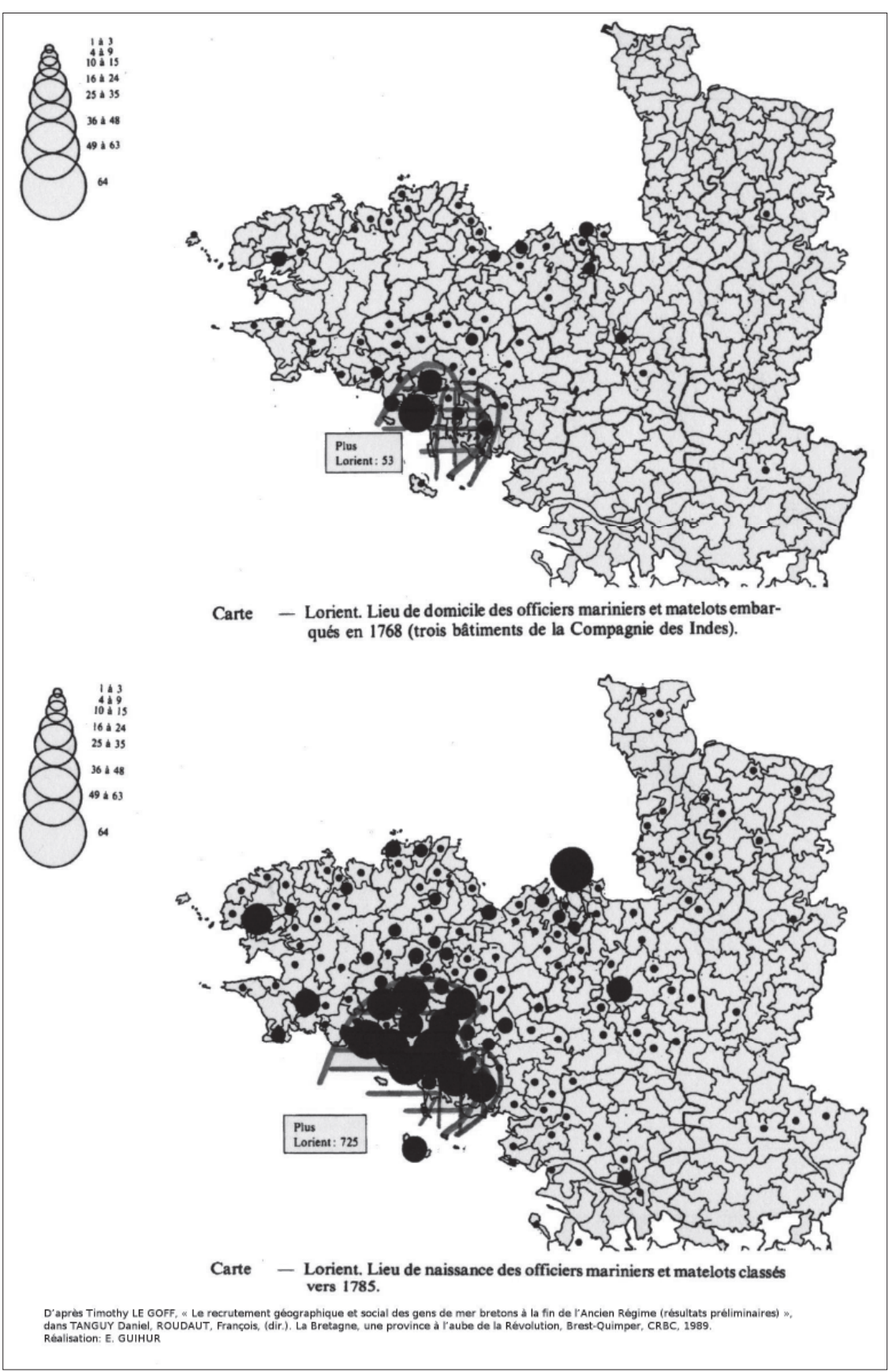




\section{RÉSUMÉ}

La Société des Missions Étrangères de Paris est constituée en 1663 par François de Laval Montmorency, François Pallu, Lambert de La Motte et Ignace Cotolendi. Elle a pour but d'évangéliser les " gentils " (païens) d'Asie par l'envoi de prêtres européens, candidats à la mission, pour former une Église locale. Dans l'esprit des fondateurs, la présence de la Société sur le continent asiatique n'est que temporaire, elle s'achèvera dès que l'Église locale pourra vivre de ses propres ressources. Les candidats à la mission, que les fondateurs souhaitent déjà ordonnés prêtres, expérimentés et de santé solide, sont assez peu nombreux entre 1663 et 1790 , un peu moins de trois cents au total. Sept prêtres du diocèse de Vannes sont intégrés à la Société et embarquent à destination de l'Asie. Comment ces hommes sont-ils entrés en contact avec cette organisation et avec la mission en Asie? Il semblerait que les origines sociales interviennent assez peu dans ce choix. De même les éventuelles relations avec des éléments du commerce asiatique lorientais ne constituent pas un critère déterminant. Les rencontres sont en revanche fondamentales : rencontres effectuées au collège, au séminaire ou en faculté de théologie, avec des étudiants ou avec des professeurs. À la lecture de la correspondance de ces sept missionnaires vannetais, un homme se distingue par sa position de destinataire privilégié : un certain M. Grinne. Ancien régent puis directeur du collège, il est souvent évoqué comme un père par ces jeunes prêtres. Ce personnage constitue le lien entre ces différents missionnaires vannetais engagés dans l'aventure d'évangélisation de l'Asie et ayant quitté l'Europe entre 1763 et 1788.

\section{ABSTRACT}

The Société des Missions Etrangères de Paris was founded in 1663 by François de Laval Montmorency, François Pallu, Lambert de La Motte and Ignace Cotolendi. It aimed at evangelizing the non-Christian Asian people by sending European priests applying for a position within the mission, the final objective being that of forming a local church. In the spirit of the founders, the presence of the Society in Asia was only temporary and its members would eventually leave the continent when the local church could evangelize by itself. The postulants, whom the founders expect to be already ordained as priests, experienced and in good health, were few between 1663 et 1790 (slightly less than 300). Seven priests from the Diocese of Vannes joined this society and sail to Asia. How did these priests come in contact with this organisation and with the Asian mission? Social origins seem to have played only an insignificant part in making the decision. At the same time, the contacts with elements of Lorient's Asian trade was not a determining factor. However, meetings with students and professors in high school, seminary or theology college were essential. Mr. Grinne appears as a regular addressee in the letters from the seven the Vannes missionaries. He was a former regent, then director of the high school in Vannes. Missionaries often called him "father". This man was the link among the missionaries from the Diocese of Vannes involved in the evangelization adventure in Asia, who left Europe between 1763 and 1788. 